\title{
The variation of English Verb Phrases in English Cookbook
}

\author{
Kadek Ayu Ekasani \\ Sekolah Tinggi Pariwisata Bali Internasional \\ Denpasar-Bali \\ ekasani@stpbi.ac.id
}

I Ketut Artawa

Universitas Udayana

Denpasar-Bali

ketut_artawa@unud.ac.id

\author{
Ida Bagus Putra Yadnya \\ English Department, \\ Faculty of Arts, Udayana University, \\ Denpasar, Bali, Indonesia
Ni Luh Ketut Mas Indrawati
Udayana University
Denpasar-Bali \\ mas.indrawati@yahoo.com
}

\begin{abstract}
This writing is entitled "The variation of English Verb Phrases in English Cookbook". The discussion is focused on the variation of English verb phrases in Cookbook. The variation of verb phrases here is some complements that follow a verb. The data used in this study is taken from English cookbook entitled “The Essential Book of Sauces \& Dressings" written by Murdoch Books and published by Periplus, Singapore. The main theory is adopted from the book "The linguistic structure of Modern English" by Brinton (2010) about the theory of verb phrases. The methods that are used to collect data are documentation and observation. The finding of the analysis shows that there are some variations of English verb phrases occur in Cookbooks, those are VP $\rightarrow \mathrm{V} \mathrm{NP}$; VP $\rightarrow \mathrm{V}$ NP PP; VP $\rightarrow \mathrm{V} \mathrm{AP;} \mathrm{VP} \rightarrow \mathrm{V}$ NP AP; $V P \rightarrow V P P ; V P \rightarrow V$ PP PP; VP $\rightarrow V$ AP PP; VP $\rightarrow V$ NP AP PP; $\mathrm{VP} \rightarrow \mathrm{V}$ NP PP PP; VP $\rightarrow$ perfect aux $V ; \mathrm{VP} \rightarrow$ passive be V PP; $\mathrm{VP} \rightarrow$ modal passive be $\mathrm{V} P P ; \mathrm{VP} \rightarrow$ passive be $\mathrm{V} P P \mathrm{PP} ; \mathrm{VP} \rightarrow$ modal passive be $V \mathrm{AP}$; VP $\rightarrow$ passive be V AP PP; VP $\rightarrow$ modal aux V NP; VP $\rightarrow$ modalaux V NP NP.
\end{abstract}

Keywords—English Verb Phrases,cookbook

\section{INTRODUCTION}

Language plays an important role in communication. Language develops with the changing times. The more often the language is used the faster its development. Even the language used often confuses the recipient of the message because its usage is sometimes underestimated.

Language is not just limited to sentences but also to a text, reading or discourse. Kridalaksana (2001, p. 231) says that discourse is the equivalent of discourse, the most complete language unit in the grammatical hierarchy which is the highest or the largest grammatical unit. Discourse can be realized in the form of compositions intact like novel, encyclopedia or recipe text. The recipe text has a very interesting sentence structure and is different from the sentence structure of the novel text or other text. According to the type of text, a recipe is a type of procedural text in which the general trait is to give instructions on how to do something through a series of actions or steps. Linguistic features use imperative sentence patterns, verbs used are active verbs (action verbs). The use of verb phrases in procedural texts such as recipe is very common and verb phrases are more likely to dominate their use in the way they are made.

The sentence structure of the cookbook text tends to use the verb phrase in initiating instructions for processing dishes. While the construction of phrases as one part in the field of syntax has a fairly complex analysis both in the structure of the phrase itself, as well as its attachment in the predicate structure. As it is known that the field of syntax is a mastery over a language that includes the ability to build phrases, clauses, sentences, and discourses derived from words. In other words syntax investigates the ins and outs of phrases, clauses, sentences and discourses (Chaer, 2007: 206).This research will look more deeply into the complexity of the verb phrases contained in English cookbook.

\section{LITERATURE REVIEW}

The structure of sentences in English is syntactically composed of two main elements, namely the subject and predicate. Subjects can be nouns or noun phrases, whereas predicates are the main verbs or verb phrases. The noun phrases and verb phrases in English are the most important phrases that cannot be omitted in sentences. English verb phrases can be divided into main verbs and verbs followed by one or more other words that serve as dependent or modifier (Carnie, 2006, p. 70).

A verb phrase is a group of words with the main verb (main verb) and auxiliaries as the central element (head). According to Carnie (2006, p. 70) "Minimally a VP consists of a single verb". This means that the minimum verb phrase consists of one verb element or can be formed according to the pattern of VP $\rightarrow \mathrm{V}$. The verb phrase in English is divided into two: simple verb phrase and complex verb phrase (Quirk, 1985 , p. 151). The simple verb phrase consists of only one verb element whereas the complex verb phrase consists of a core verb with all the complementary elements, such as auxiliary, complement and modifier consisting of adjective phrases (AdjP), noun phrases (NPs), and adverbial phrases $($ AdvP) such as the following pattern: VP $\rightarrow \mathrm{V}(\operatorname{AdjP})(\mathrm{NP})$ (AdvP). 
V PP

V PP PP

Example:

Simple Verb Phrase

a) $\mathrm{VP} \rightarrow \mathrm{V}$

Ignacious left.

$$
\mathrm{VP} \rightarrow \mathrm{V}
$$

Complex Verb Phrases

b) $\mathrm{VP} \rightarrow \mathrm{V}$ (NP) (AdvP)

Bill kissed his mother-in-law quietly.

$\mathrm{VP} \rightarrow$ [V kissed NP his mother-in-law AdvP quietly]

(Carnie, 2006, p. 70)

In this study the complexity of the structure of the verb phrase in question is a complex verb phrase where the verb phrase consists not only of a single head verb, but may comprise a verb (V) element along with all the complementary elements, such as auxiliary, complement, and modifier, consisting of an adjective phrase (AdjP), noun phrase (NP), and adverbial phrase (AdvP).

Several combinations of VGP (a combination of auxiliaries and verbs), as summarized by Quirk (1985, p. 153) as follows:

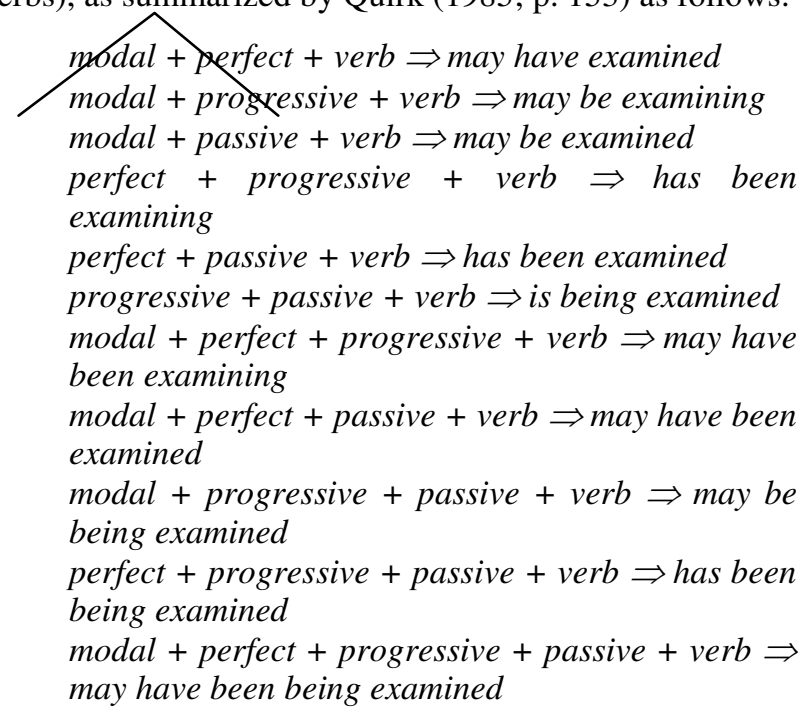

Meanwhile the category VP, the possible expansions based on Brinton are

$\begin{array}{ll}\mathrm{VP} \rightarrow \text { V NP } & \text { open a package } \\ \text { V NP NP } & \text { write a friend a letter } \\ \text { V NP PP } & \text { give an excuse to the teacher } \\ \text { V AP } & \text { feel lonely } \\ \text { V NP AP } & \text { make the dog angry }\end{array}$

(Brinton, 2010, p. 211)

Mohammadpour (2012) in his research entitled Examination of Verb and Verb Phrase in Boier Ahmadi Lori Based on X-bar Syntax. The study discusses the simple sentence analysis of the Boier Ahmadi Lori language that is one of the Indo-Iranian languages. The simple sentence structure spoken by Lori speakers is recorded and analyzed for verbs and verbs using the X-bar theory. The X-bar theory used in the study was the X-bar theory of Radford (1988). The results of the study found that through the theory of X-bar can describe the structure of verbs and verb phrases in Lori, although not as a whole can be explained through this theory, ass in the complement and adjunct elements in the structure of the verb phrase.

The verb phrase in this language belongs to a pro-drop language that removes the noun as the subject so that the verb phrase can represent the whole sentence by giving a sign of a person or a number. For example in the following sentence:

/ extelaf-I1 vel ikenan /

Discrepancy-PLURAL care of

"[They] do not care of discrepancies."

\section{VP}

$\begin{array}{cc}\text { NP } & \mathrm{V} \\ \text { extelaf } \mathbf{1} & \text { vel ikenan }\end{array}$

It is clear that the word extelaf $\mathbf{1}$ as a direct object is dominated by the verb phrase.

This research has contribution from the use of X-bar theory which can more fully illustrate the complex structure of verb phrases in cookbook. So it will be found the elements that can be categorized in accordance with the structure and verb phrase or not, as in the results of the previous research.

Other research is from Budasi (2012) entitled Contrastive Analysis of Verb Phrases in English and Bali Language Basic Sentences. The study discusses the differences and similarities of the structure of verb phrases in English and Balinese language. The theory used is the theory of Kartawinata (2010) about contrastive analysis. The data comes from interviews with native Balinese speakers in the form of some simple sentences; while for English data is taken from an English textbook. The study used the structure of the English verb phrase as a model that conforms to the construction of the Balinese verbal phrase.

The study looked at differences in verb phrase construction in English and Balinese language especially in simple sentences, positive sentences, negative sentences, and 
interrogative sentences. The structure of the English verb phrase in simple sentences can be formulated as follows:

$$
\mathrm{VP} \rightarrow\left\{\begin{array}{c}
\mathrm{V} \\
\mathrm{V}+\mathrm{NP} \\
\mathrm{V}+\mathrm{AP} \\
\mathrm{V}+\operatorname{Adjp}
\end{array}\right\}
$$

Simple sentence pattern of Balinese language generally consists of elements of NP + Predicate. Predicate here can be from elements of NP, AP, or AdjP. The structure of the Balinese verb phrase in simple sentences can also be found as in the example of Ia mebalih TV sebilang peteng (He watches TV every night), so it can be formulated as follows:

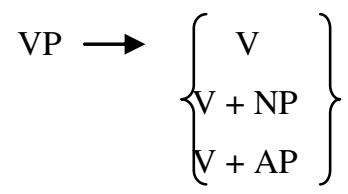

So it can be said that the structure of verb phrases in both languages has the same form. The structure of the verb phrase in the positive sentence as described in the simple sentence has the same form; the difference is only in the use of the auxiliary where the Balinese language does not have it. Construction of a negative sentence in English containing verbs requires the presence of an auxiliary verb (Aux) and a negative marker (Neg). Furthermore, the do, does, did elements which are auxiliary verbs (Aux) and Neg notes are not elements used in the Balinese language in the preparation of constructions of negative sentences. Construction of Balinese negative sentences is composed only by adding sing (not) words to verbs. The construction of interrogative sentences in English is formed by the conversion of positive sentence constructs into interrogative forms. Whereas in the Balinese language, the conversion in this way does not exist, simply adding the word 'whether' to the declarative sentence and turning the declarative intonation into an interrogative intonation, as in the example Ia melajah ditu? (He studies there?).

\section{METHODOLOGY}

This research is based on qualitative research where the data studied is qualitative data in the form of the phrase that comes from the cookbook. The type of data used in this study is qualitative data, namely in the form of the verb phrase in the cookbook. The data source used in this study is an English recipe book entitled "The Essential Book of Sauces \& Dressings" from Murdoch Books published by Periplus, Singapore.

This data source is chosen because this recipe book is an edition that has been widely used by students majoring in culinary or practitioners in the culinary field as a reference for making cooking opener and closing. In addition, the selection of cookbooks recalled western culture tends to use sauce and dressing as a basic ingredient and complementary dishes that are important because it can enhance the taste of a food. The cuisine served at the restaurant at the hotel usually prefer more continental cuisine so that the students of the department of culinary should know the making of this dressing and dressing. This is what makes researchers choose this cookbook as a corpus of data in this study. Meanwhile, the secondary data source is a literature review used in this study to support and refine the analysis of the primary data.

Methods of data collection are a method of documentation and observation. In this research the formal method is used to present the results of data analysis in tabular form, while the informal method is used to describe the research findings, that is how the complexity of verb phrases in the English cookbook.

\section{DISCUSSION}

The variation of English verb phrases in cookbook can be classified as complex verb phrases because the verb phrases consist of one or more modifiers accompanying the head verb and several phrase elements. In this study, we discussed the complex verb phrase from two points of view, i.e. verb phrase consisting of several other phrase elements or modifier accompanying the head andthe verb phrase consists of one head with several auxiliaries that accompany the head.

1. $\mathrm{VP} \rightarrow \mathrm{V} \mathrm{NP}$

Data 1 :Add the yolks (p.2)

Data 2 :Add flour and sugar. (p.6)

The complement of the verb here is a noun phrase, the yolks and flour and sugar,functioning as a direct object. While traditionally, a direct object is defined as the person or thing affected by theaction of the verb.

2. $\mathrm{VP} \rightarrow \mathrm{V}$ NP PP

Data 3 :Place the egg whites in a small dry bowl. (p.9)

Data 4 :Place the egg yolks, lemon juices and pepper in a blender. (p.9)

The next expansion of verb phrase is followed by noun phrase and prepositional phrase. The verb phrase in data 3 is place followed by noun phrase the egg whites and prepositional phrase in a small dry bowl. While in data 4 the verb place is followed by noun phrase the egg yolks, lemon juices and pepper and preposition phrase in a blender.The function of NP here are as a direct object and the PP as an object complement. It is not possible to delete the object complement because it will change the meaning of the sentence.

\section{3. $\mathrm{VP} \rightarrow \mathrm{V}$ AP}

Data 5 :Serve immediately. (p.52)

Data 6 :Serve warm (p.55) 
The complement of the verb here is Adverbial phrase, functioned as subject complement. A subject complement here characterizes the subject, although the subject of the sentences in data 5 and 6 are omitted. But it expresses a resulting state of the subject, that is the food.

\section{4. $\mathrm{VP} \rightarrow \mathrm{V} \mathrm{NP}$ AP}

Data 7 :Add wine gradually. (p.6)

Data 8 :Add the stock gradually. (p.17)

The verb phrase in data 7 and data 8 are followed by the noun phrase and also the adverbial phrase. In data 7 the expansion of the verb phrase is Verb (add) - NP (wine) - AP (gradually), and in data 8 the expansion of the verb phrase is Verb (add) - NP (the stock) - AP (gradually). Thefunction of the first NP is a direct object, the second AP is functioned as an object complement.

\section{5. $\mathrm{VP} \rightarrow \mathrm{VPP}$}

Data 9 :Stir in cream (p.6)

Data 10 :Bring to boil. (p.6)

The fifth expansion of the verb is followed by Prepositional Phrase. The prepositional phrase here is functioned as a subject complement because characterizes the subject. As same as the data 5 and 6 the subject of the sentences is omitted, but the complement of the verb phrase identifies the subject. In data 9 the expansion of the verb phrase is Verb (stir) - PP (in cream), and in data 10 the expansion of the verb phrase is Verb (bring) - PP (to boil).

\section{6. $\mathrm{VP} \rightarrow \mathrm{V}$ PP PP}

\section{Data 11 :Reheat in a pan over medium heat (p.13)}

Data 12 :Serve at room temperature with turkey, chicken or pork. (p.17)

The next expansion of verb phrase is followed by double prepositional phrase. The expansion of verb phrase in data 11 is Verb (reheat) - PP (in a pan) - PP (over medium heat) and in data 12 the expansion of verb phrase is Verb (serve) - PP (at room temperature) and PP (with turkey, chicken or pork). According to Brinton (2010, p. 210) this expansion is categorized into diprepositional verb (diprep).

\section{7. $\mathrm{VP} \rightarrow \mathrm{V}$ AP PP}

Data 13 :Simmer gently for 10 minutes. (p.2)

Data 14 :Serve warm over vegetables, spring rolls, panfried chicken or deep-fried fish. (p.14)

The expansion of verb phrase in data 13 is Verb (simmer) - AP (gently) - PP (for 10 minutes) and in data 14 the expansion is Verb (serve) - AP (warm) - PP (over vegetables, spring rolls, pan-fried chicken or deep-fried fish). Both phrases are functioned as subject complement that identifies the subject, the food.

\section{8. $\mathrm{VP} \rightarrow \mathrm{V}$ NP AP PP}

Data 15 :Heat the butter slowly in a pan. (p.9)

Data 16 :Bring the mixture slowly to the boil. (p.40)

The expansion of verb phrase in data 15 is Verb (heat) NP (the butter) - AP (slowly) - PP (in a pan) and in data 16 the expansion is Verb (bring) - NP (the mixture) - AP (slowly) - PP (to the boil). The first NP is functioned as direct object, while AP and PP functioned as object complement.

\section{9. $\mathrm{VP} \rightarrow \mathrm{V}$ NP PP PP}

Data 17 :Bring the mixture to the boil over medium heat. (p.17)

Data 18 :Whisk the yolks and sugar in a bowl for 2 minutes. (p.55)

The expansion of verb phrase in data 17 is Verb (bring) NP (the mixture) - PP (to the boil) - PP (over medium heat) and in data 18 the expansion is Verb (whisk) - NP (the yolks and sugar) - PP (in a bowl) - PP (for 2 minutes). As same as the previous expansion of the verb phrase $\{\mathrm{V}$ NP AP PP $\}$, in data 17 and 18, the first NP is also functioned as direct object, while diprep functioned as object complement

Complex Verb phrase is not only consisted by other complements but also included some specifiers of the verb or we called them auxiliary verbs, the primary auxiliaries (have and $b e$ ), the dummy auxiliary $d o$, and the modal auxiliaries (will, can, shall, may, and must) as well as a number of phrasal equivalents and borderline auxiliaries (e.g. dare, need). Here are some expansions of verb phrase which contained auxiliary verbs and some complements.

\section{0. $\mathrm{VP} \rightarrow$ aux V}

Data 19 .. . has melted (p.56)

Data 20 :. . has dissolved (p.56)

The expansion of verb phrase in data 19 and 20 is perfect auxiliary Verb (has) - Verb(melted, dissolved). In verb phrase Perfect is optional, followed by a main verb in the form of perfect participle.

\section{VP $\rightarrow$ aux V PP}

\section{Data 21 :are whisked in the machine (p.58)}

In data 21, the expansion of verb phrase is passive auxiliary verb (are) - verb in the form of past (whisked) prepositional phrase (in the machine). 
12. VP $\rightarrow$ aux V PP

Data 22 :may also be made in the food processor (p.59)

All of the specifiers of the verb are grouped together under the category of auxiliary (Aux), which is expanded in data 22, modal (may) - passive (be) - Verb (made) and added by the prepositional phrase (in the food processor).

\section{VP $\rightarrow$ aux V PP PP}

Data 23 :are whisked with the water in a pan (p.58)

The same as data 22, in data 23 the verb phrase consist of some specifiers that are group together under the category of auxiliary and followed by some complements, passive be (are) - verb (whisked) - prepositional phrase (with the water) prepositional phrase (in a pan).

\section{VP $\rightarrow$ aux V AP}

Data 24 :can be prepared ahead. (p.9)

Data 25 :must be followed closely (p.58)

For data 24 and 25 , the verb phrase is the expansion from some specifiers that are group together under the category of auxiliary and followed by adverbial phrase. The expansion is modal - passive - verb - AP.

\section{VP $\rightarrow$ aux V AP PP}

Data 26 :is added very slowly to the egg yolks(p.58)

Data 27 :is added a little at a time (p.58)

The next expansion of verb phrase in data 26 and 27 is passive auxiliary- verb - AP - PP.

\section{VP $\rightarrow$ aux V NP}

Data 28 :must use an aluminium pan (p.58)

Data 29 : will discolour the sauce (p.58)

The expansion of verb phrase in data 28 and 29 is modal auxiliary - verb - NP. In data 28, the expansion of verb phrase is modal auxiliary (must) - verb (use) - NP (an aluminium pan) and in data 29 the expansion is modal auxiliary (will) verb (discolour) - NP (the sauce).

\section{VP $\rightarrow$ auxV NP NP}

\section{Data 30 :will stop the gravy becoming lumpy (p.5)}

The last expansion of verb phrase with some auxiliaries is modal - verb - NP - NP. The same as in data 28 and 29, in data 30 the verb phrase is consisted of modal auxiliary (will) verb (stop) - NP (the gravy) - and added also by the NP (becoming lumpy) function as object complement.

\section{CONCLUSION}

There are 17 possible expansions for the category VP occur in cookbook. Those expansion of complex verb phrase can be seen from verb phrase consisting of several other phrase elements or modifier accompanying the head and the verb phrase consists of one head with several auxiliaries that accompany the head, as follows.
1. $\mathrm{VP} \rightarrow \mathrm{V} \mathrm{NP}$
2. $\mathrm{VP} \rightarrow \mathrm{V} \mathrm{NP} \mathrm{PP}$
3. $\mathrm{VP} \rightarrow \mathrm{VAP}$
4. $\mathrm{VP} \rightarrow \mathrm{V} \mathrm{NP}$ AP
5. $\mathrm{VP} \rightarrow \mathrm{V}$ PP
6. VP $\rightarrow$ V PP PP
7. $\mathrm{VP} \rightarrow \mathrm{V}$ AP PP
8. $\mathrm{VP} \rightarrow \mathrm{V}$ NP AP PP
9. VP $\rightarrow$ V NP PP PP
10. $\mathrm{VP} \rightarrow$ perfect aux $\mathrm{V}$
11. VP $\rightarrow$ passive be V PP
12. VP $\rightarrow$ modal passive be V PP
13. VP $\rightarrow$ passive be V PP PP
14. $\mathrm{VP} \rightarrow$ modal passive be $\mathrm{V}$ AP
15. VP $\rightarrow$ passive be V AP PP
16. VP $\rightarrow$ modal V NP
17. VP $\rightarrow$ modal V NP NP

\section{References}

Brinton, L. J. \& Donna M. B. (2010). The linguistic structure of Modern English, (2nd ed.) Amsterdam: John Benjamins Publishing Company.

Budasi, I.G. (2012). Contrastive analysis of verb phrases in English and Bali language basic sentences. PRASI, 8. Retrieved from https://ejournal.undiksha.ac.id/ index.php/PRASI/article/view/433

Carnie, A. (2006). Syntax: A generative introduction Second Edition. Australia: Blackwell Publishing.

Chaer, A. (2007). Tata bahasa praktis bahasa Indonesia. Jakarta: Rineka Cipta.

Kridalaksana, H. (2001). Kamus Linguistik. Jakarta: Gramedia Pustaka Utama.

Lowery, B., Brodhust, W., Goggin, W., Earl, M. (1996). The essential book of sauces and dressings. Singapore: Periplus

Mohammadpour, F. (2012). Examination of verb and verb phrase in Boier Ahmadi Lori based on X-bar syntax. International Journal of Linguistics. 4, 509-522. doi:10.5296/ijl.v4i3.2375

Quirk, R. (1985). A comprehensive grammar of the English language. USA : Longman Inc.

Sutopo, H.B. (2006). Penelitian kualitatif : dasar teori dan terapannya dalam penelitian. Surakarta: UNS. 